\title{
Colesterol y triglicéridos como marcadores bioquímicos del estado de la enfermedad del paciente con leucemia linfocítica aguda
}

\author{
MARCO GUZMÁN ${ }^{1}$, MIGUEL SANDOVAL ${ }^{2}$ \\ ${ }^{1}$ nstituto de Enfermedades Neoplásicas. \\ ${ }^{2}$ Centro de Investigación de Bioquímica y Nutrición, Facultad de Medicina - UNMSM
}

\begin{abstract}
Resumen
O bjetivo: D eterminar la relación de los niveles séricos de colesterol y triglicéridos con el estado de respuesta al tratamiento quimioterápico de inducción de pacientes con leucemia linfocítica aguda. Material y M étodos: La muestra la conforman 25 pacientes de 2 a 18 años de edad, admitidos al Instituto de Enfermedades Neoplásicas con un diagnóstico reciente de leucemia linfocítica aguda; determinándose en ellos sus concentraciones séricas de colesterol total, colesterol-HDL, colesterol-LDL y triglicéridos, antes y después de la primera fase de la quimioterapia de inducción. Resultados: Solamente 23 pacientes respondieron al tratamiento y en ellos observamos un incremento de 19,24 \pm 4,49 mg/dL a 46,84 $\pm 15,38 \mathrm{mg} / \mathrm{dL}$ para el colesterol-HDL; y un descenso de 153,66 $\pm 36,39 \mathrm{mg} / \mathrm{dL}$ a 79,79 $\pm 34,53 \mathrm{mg} / \mathrm{dL}$ para los triglicéridos $(p<0,0001)$. Por el contrario, en los pacientes que no respondieron al tratamiento, se obtuvo un incremento de $14,64 \pm 2,46 \mathrm{mg} / \mathrm{dL}$ a $23,13 \pm 14,23 \mathrm{mg} / \mathrm{dL}$ para el colesterolHDL y un incremento de $121,88 \pm 0,56 \mathrm{mg} / \mathrm{dL}$ a $161,01 \pm 75,25 \mathrm{mg} / \mathrm{dL}$ para los triglicéridos. Conclusión: L as concentraciones séricas de colesterol y triglicéridos se relacionan con el estado de la enfermedad del paciente con leucemia linfocítica aguda. Proponemos a estos metabolitos, como marcadores bioquímicos en el seguimiento clínico de esta enfermedad.
\end{abstract}

Palabras clave: Hipocolesterolemia; lipoproteinas del colesterol HDL; lipoproteinas del colesterol LDL; Ieucemia linfocítica aguda; triglicéridos.

\section{Cholesterol and triglycerides as biochemical markers of the state of the patient's illness with acute lymphocytic leukemia}

\section{Abstract}

Objective: To determine the relationship of cholesterol and triglycerides serum levels with the response to induction chemotherapy treatment in patients with acute lymphocytic leukemia. $M$ aterial and M ethods: The sample consisted in 25 patients 2 through 18 years-old admitted to the N eoplasia Diseases Institute with a recent diagnosis of acute lymphocytic leukemia in whom serum concentrations of total cholesterol, HDL-cholesterol, LDL-cholesterol and triglycerides were determine, before and after the first phase of the induction chemotherapy. Results: Only 23 patients responded to the treatment and in them we observe an increment from 19,24 $\pm 4,49$ $\mathrm{mg} / \mathrm{dL}$ to $46,84 \pm 15,38 \mathrm{mg} / \mathrm{dL}$ for the HDL-cholesterol, and a descent from $153,66 \pm 36,39$ $\mathrm{mg} / \mathrm{dL}$ to $79,79 \pm 34,53 \mathrm{mg} / \mathrm{dL}$ for the triglycerides $(p<0,0001)$. On the contrary, in the patients that didn't respond to the treatment, an increment was obtained from $14,64 \pm 2,46 \mathrm{mg} /$ $\mathrm{dL}$ to $23,13 \pm 14,23 \mathrm{mg} / \mathrm{dL}$ for HDL-cholesterol and an increment from $121,88 \pm 0,56 \mathrm{mg} / \mathrm{dL}$ to $161,01 \pm 75,25 \mathrm{mg} / \mathrm{dL}$ for triglycerides. Conclusion: Serum concentrations of cholesterol and triglycerides are correlated with the state of the patient's illness with acute lymphocytic leukemia. We propose these metabolites as biochemical markers in the clinical pursuit of this illness.

Key words: Hypocholester olemia; lipoproteins, HDL, cholesterol; lipoproteins, LDL, cholesterol; leukemia, lymphocytic, acute; triglycerides. 


\section{INTRODUCCIÓN}

En las neoplasias hematológicas, como la policitemia vera, metaplasia mieloide, mieloma múltiple, leucemia mieloide, leucemia linfocítica y al gunos síndromes mieloproliferativos crónicos, se ha reportado un descenso de los nivel es séricos de colesterol total y que luego de un efectivo tratamiento de la enfermedad, retornan a sus valores normales. L as bajas concentraciones séricas de colesterol total en pacientes con neoplasias hematológicas se deberían a la disminución en los niveles de colesterol-HDL y colesterol-LDL, y estos a su vez se deberían a un descenso en sus fracciones lipoproteicas HDL y $L D L$, respectivamente $\left({ }^{1-3}\right)$.

Baroni y col. evaluaron el metabolismo del colesterol en pacientes adultos con leucemia linfocítica aguda ( LLA) y leucemia mieloide aguda ( L M A ), tanto en la etapa diagnóstica como en la etapa de remisión completa de la enfermedad. En la etapa diagnóstica hallaron niveles séricos disminuidos de colesterol total, colesterol-HDL y colesterol-LDL, además de una marcada hipertrigliceridemia en ambos tipos de enfermedad. En los pacientes con L LA que respondieron a la quimioterapia, se observó un incremento significativo de colesterol total, colesterol-HDL y apoproteína A -I (apoA-I), con una disminución de los triglicéridos; mientras que, en los pacientes con $L M A$ que respondieron a la quimioterapia, se obtuvo un incremento significativo en los niveles de colesterol total, colesterol-LDL y apoproteína B. No se observó ninguna variación en el grupo de pacientes que no respondieron satisfactoriamente a la quimioterapia. Este estudio demuestra un comportamiento diferente de las fracciones lipoproteicas del colesterol en ambos tipos de enfermedad, el colesterol-LDL en Ia LMA y el colesterol-HDL en la LLA ${ }^{(1)}$.

Estudios practicados in vitro e in vivo sobre los leucocitos de pacientes con LM A, permitieron determinar en éstos una mayor captación de LD L (lipoproteína de baja densidad), debido a una mayor actividad de los receptores de membrana para LDL $\left(^{4-11}\right)$. Estos estudios también fueron practicados sobre los leucocitos de pacientes con L LA en donde se observó una captación normal de $L D L$, similar a la de leucocitos de pacientes sanos. Entonces en la LLA existirían otros mecanismos que justifiquen un descenso del colesterol, diferente al que se presenta en los pacientes con L M A $\left({ }^{6,12-15}\right)$.

El descenso marcado de colesterol-HDL en pacientes con LLA y su notable incremento durante la etapa de remisión completa, obedecería a la variación en la concentración de HDL (lipoproteína de alta densidad) y que a su vez se relacionaría con una respuesta de fase aguda $\left({ }^{16}\right)$.

Los estímulos que desencadenan la respuesta de fase aguda son múltiples y todos ellos comparten la capacidad de estimular a los macrófagos. Los productos resultantes de la activación de los macrófagos, como la IL -1, IL 6 y el factor de necrosis tumoral, están implicados en el inicio de la síntesis de proteínas de fase aguda. La mayoría de las proteínas de fase aguda son sintetizadas por los hepatocitos, y durante los procesos inflamatorios incrementan su concentración sérica hasta 1000 veces; siendo las más importantes la proteína $C$ reactiva $(P C R)$ y la proteína SA A . L a proteína SA A se caracteriza por incorporarse a las HDL y desplazar a sus principales apoproteínas $A-I$ y $A-I I$, teniendo como consecuencia una aceleración del catabolismo de las HDL y su disminución sérica durante los procesos inflamatorios $\left({ }^{17-20}\right)$.

La proteína SAA es una apoproteína denominada también apoSA A y es similar a la apoA -I $\left({ }^{21}\right)$. D urante la respuesta de fase aguda, las HDL son ricas en apoSAA, Ilegando a representar el $50 \%$ del total de apolipoproteínas de las HDL en el pico de la respuesta de fase aguda. Por el contrario, en condiciones normales, las concentraciones de apoSA A dentro de las HDL son muy bajas (22). Durante la respuesta de fase aguda, se ha demostrado una correlación inversa entre el contenido de apoAI, apoA-II y colesterol-HDL con las concentraciones séricas de apoSAA $\left({ }^{23,24}\right)$. También se ha observado un catabolismo acelerado de las partículas de HDL ricas en apoSA A $(25,26)$. En un modelo experimental, se demostró como las HDL ricas en apoSA A eran captadas por los macrófagos mediante un proceso de endocitosis $\left({ }^{27}\right)$. Estos resultados apoyan el mecanismo de desplazamiento de las apoproteínas A-I y A-II por la apoSAA para explicar el 
aumento del catabolismo de las HDL y en consecuencia un descenso de los niveles de séricos de colesterol-HDL.

$L$ a respuesta de fase aguda también tiene sus efectos sobre los triglicéridos, induciendo un incremento en sus concentraciones séricas al estimular la lipogénesis hepática y la producción de VLDL (lipoproteína de muy baja densidad), así como la síntesis hepática y movilización periférica de los ácidos grasos libres $\left({ }^{28}\right)$. Los ácidos grasos libres movilizados serán reesterificados hasta triglicéridos en el hígado y luego vueltos a secretar, contribuyendo al incremento de los nivel es plasmáticos de V LDL. A este mecanismo se le ha dado el nombre de "ciclo fútil", en el cual los ácidos grasos libres son transportados desde el tejido adiposo hasta el hígado y devueltos nuevamente al tejido adiposo, sin haber sido utilizados como fuente de energía (28-30).

La relación respuesta de fase aguda y neoplasias hematológicas, ha sido demostrada en una observación realizada sobre un paciente con linfoma de células $B$. En él se encontró niveles muy descendidos de colesterol-HDL (3 $\mathrm{mg} / \mathrm{dL})$ y apo $\mathrm{A}-\mathrm{I}(17,4 \mathrm{mg} / \mathrm{dL})$, además concentraciones elevadas de triglicéridos (272 $\mathrm{mg} / \mathrm{dL})$. Junto con ellos, se determinó concentraciones séricas el evadas de proteína $C$ reactiva y apoSA A. A estos resultados se añadía un análisis de gradiente de densidad de las lipoproteínas, que demostraba la presencia casi nula de partículas HDL en este individuo $\left({ }^{31}\right)$.

El objetivo del presente estudio es demostrar la relación entre los niveles séricos de colesterol y triglicéridos con el estado de respuesta al tratamiento quimioterápico de inducción en pacientes con leucemia linfocítica aguda.

\section{MATERIAL Y MÉTODOS}

El estudio es de tipo prospectivo, de corte longitudinal y analítico. La muestra la conformaron 25 pacientes (14 varones y 11 mujeres) entre 2 y 18 años, siendo admitidos al Instituto de Enfermedades Neoplásicas con un diagnóstico reciente de $L L A$ entre los meses de marzo y junio de 2002.
A cada paciente se le realizó dos determinaciones bioquímicas de colesterol total, colesterol-HDL, colesterol-LDL y triglicéridos. La primera determinación correspondió al momento del diagnóstico (antes del tratamiento); $y$, la segunda, a la fecha en que culminó la primera etapa de la fase de la quimioterapia de inducción (después del tratamiento), aproximadamente 30 días después del momento del diagnóstico y que además correspondía a la fecha de al ta de hospitalización. La información referente al diagnóstico inicial, así como, el estado de la enfermedad, finalizada la primera fase de la quimioterapia de inducción, se obtuvo a través de la revisión de historias clínicas.

L as determinaciones séricas de colesterol total, colesterol-HDL y colesterol-LDL se efectuó por métodos enzimáticos, previa precipitación de las lipoproteínas LDL y VLDL para el dosaje de colesterol-HDL; y precipitación selectiva de lipoproteína LDL (método indirecto), para el dosaje de colesterol-LDL. La determinación de triglicéridos se realizó por el método enzimático con colorimetría según Trinder.

L as muestras fueron procesadas por duplicado y empleando sueros de control. El control de calidad externo se realizó en el equipo automatizado Beckman Synchron. Los valores numéricos de los metabolitos de interés expresados en $\mathrm{mg} / \mathrm{dL}$ fueron procesados por la "Prueba de la suma de rangos de Wilcoxon", a través del programa estadístico SPSS para $W$ indows, versión 10,0 . A demás, se hizo uso de proporciones con el uso de porcentajes, para demostrar la variación de los metabolitos durante el estudio.

\section{RESULTADOS}

Sólo 23 pacientes lograron la remisión completa y en ellos se observó que el colesterol total se incrementó de $123,08 \pm 23,52 \mathrm{mg} / \mathrm{dL}$ a $155,39 \pm 36,79 \mathrm{mg} / \mathrm{dL}$, el colesterol-HDL de $19,24 \pm 4,49 \mathrm{mg} / \mathrm{dL}$ a $46,84 \pm 15,38 \mathrm{mg} / \mathrm{dL}$; el colesterol-LDL de $78,31 \pm 20,12 \mathrm{mg} / \mathrm{dL}$ a $94,27 \pm 36,08 \mathrm{mg} / \mathrm{dL}$. Los triglicéridos disminuyeron de $153,66 \pm 36,39 \mathrm{mg} / \mathrm{dL}$ a 79,79 $\pm 34,53 \mathrm{mg} / \mathrm{dL}$. En los 2 pacientes que no respondieron a la quimioterapia de inducción y 
no alcanzaron la remisión completa, se presentaron las siguientes variaciones. Un incremento de 140,45 $\pm 36,33 \mathrm{mg} / \mathrm{dL}$ a 154,94 $\pm 33,79 \mathrm{mg} / \mathrm{dL}$ para el colesterol total; de 14,64 $\pm 2,46 \mathrm{mg} / \mathrm{dL}$ a $23,13 \pm 14,23 \mathrm{mg} / \mathrm{dL}$ para el colesterol-HDL; y de $121,88 \pm 0,56 \mathrm{mg} / \mathrm{dL}$ a $161,01 \pm 75,25 \mathrm{mg} / \mathrm{dL}$ para los triglicéridos. El colesterol-LDL disminuyó de 101, $57 \pm 34,85$ $\mathrm{mg} / \mathrm{dL}$ a $73,69 \pm 11,21 \mathrm{mg} / \mathrm{dL}$.

El colesterol-HDL muestra en la primera medición, una ajustada concentración de los valores, al rededor de la mediana $(19,89 \mathrm{mg} / \mathrm{dL})$; y en la medición final, una mayor dispersión de los datos con una mediana de $44,13 \mathrm{mg} / \mathrm{dL}$ (Figura 1). Los triglicéridos antes y después del tratamiento, tiene como medianas $146,44 \mathrm{mg} /$ $\mathrm{dL}$ y $62,55 \mathrm{mg} / \mathrm{dL}$, respectivamente (F igura 2 ).

La variación del colesterol total, sus fracciones lipoproteicas y los triglicéridos, expresada en porcentajes, tanto para el grupo de pacientes que alcanzaron y no alcanzaron la remisión completa, se muestra en la Figura 3.

\section{DISCUSIÓN}

El incremento de colesterol-HDL de 153,92\% y su $p<0,0001$ en los pacientes que Iograron la remisión, se correlaciona con los hallazgos descritos por Baroni S. y cols. Ellos obtuvieron en su muestra un incremento de 28,28 $\pm 5,46$ a $60,45 \pm 15,60 \mathrm{mg} / \mathrm{dL}$ para el colesterol-HDL, mientras que nosotros, un incremento de 19,24 $\pm 4,49$ a 46,84 $\pm 15,38 \mathrm{mg} / \mathrm{dL}$. Considérese además que la diferencia entre ambos trabajos es el rango de edad de los pacientes que conforman la muestra; en nuestro estudio es de 2 a 18 años, mientras que en el otro, de 15 a 60 años. N uestros resultados también revelan que los triglicéridos, después del colesterol-HDL, se correlaciona con el estado de respuesta y no respuesta al tratamiento, sufriendo una notable caída de $-48,08 \%$ en los pacientes que respondieron al tratamiento, y su incremento de $32,25 \%$ en los pacientes que no respondieron al tratamiento.

Nos preguntamos si la leucemia linfocítica aguda ocasiona una respuesta de fase aguda y un descenso de los niveles de lipoproteína HDL, apoA-I y colesterol-HDL, ¿por qué la bibliografía demuestra que no sucede lo mismo con la leucemia mieloide aguda? Si también ella corresponde a una neoplasia hematológica y podría desencadenar una respuesta de fase aguda; y, más bien, se ha demostrado en ella un descenso de los niveles de lipoproteína $L D L$, apoB y colesterol-LDL. La respuesta a ello podría deberse a una captación muy incrementada de lipoproteína LDL por parte de los blastos de estirpe mieloide $(9,10,12)$.

Los resultados de nuestro estudio, especialmente el de colesterol-HDL y triglicéridos, no sólo se relacionan con el estado de respuesta y no respuesta al tratamiento, sino

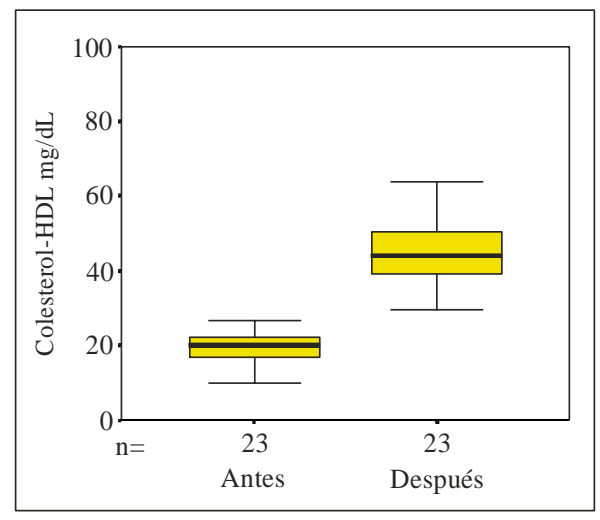

Figura 1. Colesterol-HDL antes y después de la quimioterapia de inducción en pacientes con remisión.

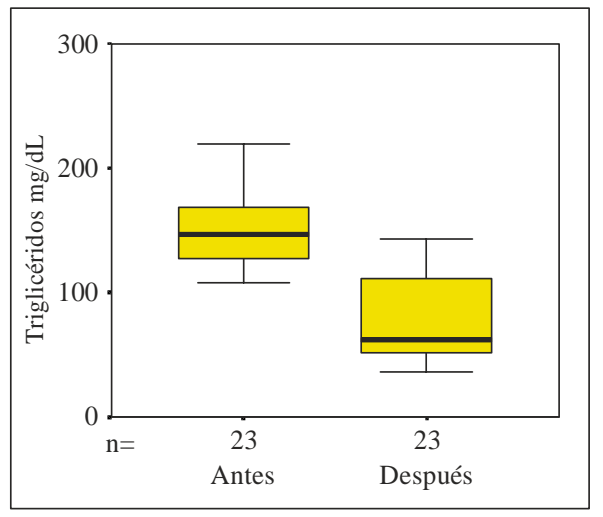

Figura 2. Triglicéridos antes y después de la quimioterapia de inducción en pacientes con remisión. 


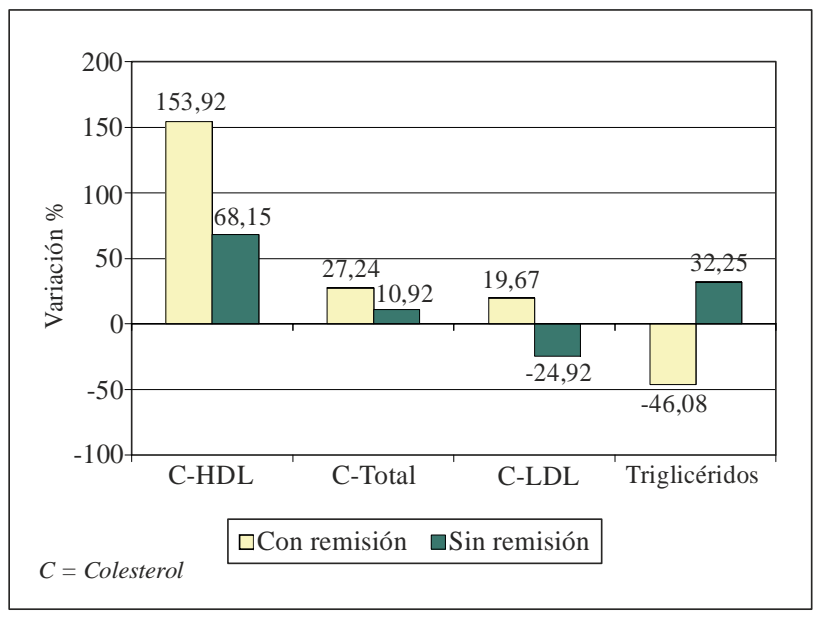

Figura 3. Variación porcentual de colesterol y triglicéridos en pacientes con LLA con y sin remisión al tratamiento quimioterápico.

también con la actividad de la enfermedad, determinada por el grado de estabilidad clínica del paciente, habiendo observado esto en los dos pacientes que no respondieron al tratamiento. El primero de ellos falleció a los 51 días de su internamiento, tiempo en el que presentó múltiples complicaciones clínicas, siendo sus concentraciones de colesterol-HDL en el día 1 , 28 y 48 , de $16,38 \mathrm{mg} / \mathrm{dL}, 13,47 \mathrm{mg} / \mathrm{dL}$ y 13,06 $\mathrm{mg} / \mathrm{dL}$, respectivamente; es decir una tendencia a la caída. Por el contrario, el segundo paciente no presentó mayores complicaciones durante su internamiento de 28 días, luego del cual se retiró con un buen grado de estabilidad clínica, aunque no logró la remisión completa. Sus concentraciones de colesterol-HDL durante los días 1,14 y 26 fueron de $12,9 \mathrm{mg} / \mathrm{dL}, 40,3 \mathrm{mg} /$ $\mathrm{dL}$ y $33,19 \mathrm{mg} / \mathrm{dL}$.

Con los resultados de nuestro trabajo y de estudios previos, podemos atribuir a la leucemia linfocítica aguda un rol desencadenante en la respuesta de fase aguda, que disminuye los niveles de colesterol total, colesterol-HDL, colesterol-LDL y aumenta los triglicéridos; y que luego de finalizada la quimioterapia de inducción, dirigida a eliminar a las células neoplásicas y llevar al paciente a un estado de remisión completa, se suprimiría la respuesta de fase aguda, con la consecuente normalización de los niveles de colesterol total, colesterol-LDL y principalmente de colesterol-HDL y triglicéridos. Ponemos énfasis en estos dos últimos metabolitos, porque han sido los que han tenido un mayor porcentaje de variación, una menor dispersión de los datos y un comportami ento más fiel con respecto al estado de remisión y no remisión completa del paciente.

Las concentraciones de colesterol, sus fracciones lipoproteicas y los triglicéridos se relacionan con el estado de la enfermedad del paciente con $L L A$ sometido a quimioterapia de inducción, tanto en aquellos que lograron y no lograron la remisión completa, por lo que proponemos a estos metabolitos como marcadores bioquímicos en el seguimiento clínico de esta enfermedad, uniéndose a otros marcadores, como son, el estudio de médula ósea y de sangre periférica; por ello, la determinación bioquímica del colesterol, sus fracciones lipoproteicas y triglicéridos podría tomarse también como una prueba pronóstica de la evolución del paciente con $L L A$. L os resultados de este trabajo y los antecedentes nos impulsan a hacer esta propuesta.

\section{AGRADE CIMIENTOS}

A la Doctora Carmen Armas Purilla por su incondicional apoyo. A sí mismo, a todo el personal de laboratorio del Servicio de Bioquímica, Hematología Especial, y de Emergencias STAT del Instituto de Enfermedades N eoplásicas.

\section{REFERENCIAS BIBLIOGRÁFICAS}

1. Baroni S, Scribano D, Zuppi C, Pagano L, Leone G, Giardina B. Prognostic relevance of lipoprotein cholesterol levels in acute lymphocytic and nonlymphocytic leukemia. A cta Haematol. 1996;96:24-8.

2. Gilbert HS, Ginsberg H, Fagerstrom R, Brown WV. Characterization of hypocholesterolemia in myeloproliferative disease. Relation to disease manifestations and activity. A m J M ed. 1981;71:595-602.

3. Lorenc J, Kozak-M ichalowska I, Polkowska-Kulesza E. Disorders of lipid and lipoprotein metabolism in patients with chronic lymphocytic leukemia. I. Preliminary evaluation of lipemia and HDL fractions in various stages of the disease. Przegl Lek. 1989;46:713-8.

4. Fazio S, Hasty A, Carter K, M urray A, Price J, Linton M. L eukocyte low density lipoprotein receptor ( $L D L-R$ ) does not contribute to $L D L$ clearance in vivo: bone marrow 
transplantation studies in the mouse. J Lipid Res. 1997;38:391-400.

5. Peterson C, Vitols S, Rudling M, Blomgren $H$, Edsmyr F, Skoog L. Hypocholesterolemia in cancer patients may be caused by elevated LDL receptor activities in malignant cells. M ed Oncol Tumor Pharmacother. 1985;2:143-7.

6. Rudling $M$, Gåfvels $M$, Parini P, Gahrton G, A ngelin B. $L$ ipoprotein receptors in acute myelogenous leukemia. A m J Pathol.1998; 153: 1923-35.

7. Tatidis $L, G$ ruber $A, V$ itols $S$. D ecreased feedback regulation of low density lipoprotein receptor activity by sterols in leukemic cells from patients with acute myelogenous leukemia. J Lipid Res. 1997;38:2436-45.

8. Vitols S, Gahrton G, Peterson C. Significance of the lowdensity lipoprotein $(L D L)$ receptor pathway for the in vitro accumulation of AD-32 incorporated into LDL in normal and leukemic white blood cells. Cancer Treat Rev. 1984;68:515-20.

9. Vitols S, Gahrton G, Ost A, Peterson C. Elevated low density lipoprotein receptor activity in leukemic cells with monocytic differentiation. Blood. 1984;63:1186-93.

10. Vitols S, Gahrton G, Bjorkholm M, Peterson C. $\mathrm{Hypocholesterolaemia} \mathrm{in} \mathrm{malignancy} \mathrm{due} \mathrm{to} \mathrm{elevated} \mathrm{low-}$ density-lipoprotein-receptor activity in tumour cells: evidence from studies in patients with leukaemia. L ancet. 1985;2:1150-4.

11. Vitols S, Angelin B, Ericsson S, Gahrton G, Juliusson G, $M$ asquelier $M$, et al. U ptake of low density lipoproteins by human leukemic cells in vivo: R elation to plasma lipoprotein levels and possible relevance for selective chemotherapy. PNAS. 1990;87:2598-602.

12. Budd D, Ginsberg H. Hypocholesterolemia and acute myelogenous leukemia. A ssociation between disease activity and plasma low-density lipoprotein cholesterol concentrations. Cancer. 1986;58:1361-5.

13. Hinnie J, O'Reilly D. Hypocholesterolaemia in hairy cell leukaemia. Br M ed J. 1995;311:1437.

14. Juliusson G, Vitols S, Liliemark J. Mechanisms behind hypocholesterolaemia in hairy cell leukaemia. $\mathrm{Br} \mathrm{M}$ ed J. 1995;311:27.

15. Juliusson G, Vitols S. Impaired low-density lipoprotein receptor activity in chronic B-lymphocytic leukaemis cells. Eur J Haematol. 1988; 40:18-24.

16. Baroni S, Scribano D, Pagano L, Zuppi C, Leone G, Giardina B. Lipids and lipoproteins in acute lymphoblastic leukaemia (ALL). L euk Res. 1994;18:643-4.

17. Cabana V, Lukens J, Rice K, H awkins T, Getz G. HDL content and composition in acute phase response in three species. J Lipid Res. 1996;37:2662-74.

18. Cabana V, Reardon C, Wei B, Lukens J, Getz G. SAA only $H D L$ formed during the acute phase response in apoA I + /+ and ApoA-I -/- mice. J Lipid Res. 1999;40:1090103.

19. Cuetzee G, Strachan A, V an Der W esthuyzen D, Hops H, J eenah $M$, De B eer F. Serum amyloid A -containing human high density lipoprotein 3: density, size and apolipoproteins composition. J Biol Chem. 1986;26:9644-51.
20. Lindshorst $E$, Y oung D, Bagshaw W, H yland M, K isilevsky $R$. A cute inflammation, acute phase serum amyloid $A$ and cholesterol metabolism in the mouse. Biochim Biophys A cta. 1997; 1339: 143-54.

21. E rickson N, Benditt $E$. Isolation and characterization of the amyloid-related apoprotein (SA A) from human high density lipoprotein. PNAS. 1980;77:6860-4.

22. $V$ an $L$ enten $B$, Hama $S$, De B eer $F$, Stafforim D, M clntyre $T$, Prescott $S$. Anti-inflammatory HDL becomes proinflammatory during the acute phase response. Loss of protective effect of $H D L$ against $L D L$ oxidation in aortic wall cell cocultures. J Clin Invest. 1995;96:2758-67.

23. Cabana V, Siegel J, Sabesin S. Effects of the acute phase response on the concentration and density distribution of plasma lipids and apolipoproteins. J Lipid Res. 1989;30: 39-49.

24. Kumon Y, Suchiro T, Ikeda I, Y oshida K, Hashimoto K, Ohno F. Influence of serum amyloid A protein on highdensity lipoprotein in chronic inflammatory disease. Clin Biochem. 1993; 26:505-11.

25. Bausserman L, Herbert P, Rodger R, Nicolosi R. Rapid clearance of serum amyloid A from high-density lipoproteins. Biochim Biophys Acta. 1984;792:186-91.

26. Parks J, Rudell L. M etabolism of the serum amyloid A proteins (SA A) in high density lipoproteins and chylomicrons of nonhuman primates (vervet monkeys). A m J Pathol. 1983; 112:243-9.

27. Rocken C, Kisilevsky R. Comparison of the binding and endocytosis of high-density lipoprotein from heal thy ( $\mathrm{HDL}$ ) and inflamed (HDL-SAA) donors by murine macrophages of four different mouse strains. V irchows A rch. 1998;432: 547-55.

28. Grunfeld C, F eingold K. M etabolic disturbances and wasting in the acquired immunodeficiency syndrome. $\mathrm{N}$ Engl J M ed. 1992;327:329-35.

29. Grunfeld C, Pang M, Doerrler W. Lipids, lipoprotein, triglyceride clearance and cytokines in human immunodeficiency virus infection and the acquired immunodeficiency syndrome 1992. J Clin Endocrinol M etab. 1992; 74: 1045-52.

30. Gutierrez R, Campa A, Shor-Posner G, Baum M. Estado del metabolismo de los lípidos en la infección por VIH-1 y SIDA. Diagnóstico. 1998;37:336-43.

31. Blackman J, Cabana V, M azzone T. The acute phase response and associated lipoprotein abnormalities accompanying lymphoma. J Intern M ed. 1993;233:201-4.

Correspondencia:

Lic. T.M. Miguel H. Sandoval Vegas

Centro de Investigación de Bioquímica y Nutrición

Facultad de Medicina - UNMSM

Av. Grau 755. Lima 1, Perú

Correo-e: msandovalv@sanfer.unmsm.edu.pe 